\title{
Percepción de jóvenes rurales frente al ecoturismo en el Centro Ambiental Chimayoy, Municipio de Pasto, Colombia
}

\author{
Gloria C. Luna-Cabrera, Alejandra Narváez-Romo y Ángela A. Molina-Moreno \\ ,Universidad de Nariño, Facultad de Ciencias Agrícolas, Programa de Ingeniería Agroforestal. Calle 18 Carrera 50 \\ Ciudadela Universitaria Torobajo, Pasto, Nariño, Colombia. (correo-e: grupopifil@gmail.com; nrale.14@gmail.com; \\ agrofore9@hotmail.com)
}

Recibido Ago. 29, 2019; Aceptado Oct. 22, 2019; Versión final Dic. 20, 2019, Publicado Abr. 2020

\begin{abstract}
Resumen
La presente investigación tuvo como objetivo identificar la percepción de jóvenes rurales frente al ecoturismo. Mediante investigación cualitativa, se diseñó y aplicó encuestas y herramientas de análisis situacional con muestreo de tipo aleatorio simple. Se evidenció que los jóvenes rurales consideran como principales problemáticas el uso inadecuado de los recursos naturales (62\%), la falta de compromiso de los jóvenes por el cuidado de los recursos naturales (73\%), y el poco conocimiento sobre ecoturismo (78\%). También destacan como actividades ecoturísticas los talleres de educación ambiental y caminata guiada. Se identificó como debilidad la mínima integración de la comunidad, su fortaleza el talento, y como oportunidades el apoyo institucional. Esto permite generar estrategias de formación en formulación de proyectos, educación ambiental y ecoturismo. Se concluye que los jóvenes rurales tienen apropiación por su territorio, constituyendo una oportunidad para la estructuración de proyectos de emprendimiento turístico.
\end{abstract}

\section{Perception of rural youth with respect of ecotourism in the Chimayoy Environmental Center, Municipality of Pasto, Colombia}

\begin{abstract}
The purpose of this research was to identify the perception of rural young people about ecotourism. Through qualitative research, surveys and situational analysis with simple random sampling were designed and applied. The rural youth consider as main problems the inadequate use of natural resources $(62 \%)$, the lack of commitment of youth for the care of natural resources $(73 \%)$, and little knowledge about ecotourism (78\%). Also, environmental education workshops and guided walks are highlighted as ecotourism activities. The minimal integration of the community was identified as a weakness, the talent as a strength, and institutional support were identified as an opportunity. This allows the generation of training strategies in project formulation, environmental education and ecotourism. It is concluded that the rural youth have appropriation for their territory, constituting an opportunity for the structuring of projects of tourist enterprise
\end{abstract}

Keywords: environmental management; environmental education; alternative tourism; rural education; rurality 


\section{INTRODUCCIÓN}

Los jóvenes rurales en América Latina son un grupo importante y un recurso estratégico para el desarrollo de los territorios rurales, aún más la tendencia al envejecimiento de la población rural posiciona a las nuevas generaciones en un rol privilegiado (Heidkamp y Morrisey, 2019). Lo juvenil requiere por lo tanto su comprensión como un concepto lleno de contenido dentro de un contexto histórico y sociocultural, y por ende la condición de ser joven, posee una simbolización cultural con variaciones fundamentales en el tiempo (Alvarado et al., 2009). La jerarquía sobrepone lo urbano a lo rural y continúa reproduciéndose entre los habitantes del campo e influenciando sobre la cultura de éstas; en el medio rural la representación que asocia la vida y prosperidad en la ciudad, ha promulgado que vivir y trabajar en el campo es de menor valía (Neleman y Castro, 2016).

Cómo lo expresa Batra (2013), el modo de producción capitalista ha generado una sistemática destrucción de la naturaleza y es causa de la crisis ambiental porque además de ejercer exclusión sobre los más pobres les limita su posibilidad de trabajo al condicionar la inversión a la ganancia. El homo oeconomicus de que habla Pigem (2013) es un sujeto individualista ligado a abstracciones, desvinculado de sentimientos, de la naturaleza y del bien común, el modelo del homo oeconomicus se basa en un crecimiento económico ilimitado sustentado en el consumo de una naturaleza limitada, Leff (2006), nada más alejado de los procesos de diálogo de saberes y organización comunitaria. Sin embargo el ecoturismo que visto desde una perspectiva etnoecológica al decir de Toledo (1990), reivindica e incorpora la cosmovisión de los pueblos originarios y campesinos habitantes de muchos de los territorios susceptibles de integrarse a modelos de turismo sostenible puede representar una alternativa al modelo extractivo imperante "porque respetar al mundo natural como se debiera respetar al prójimo, es reconocerlo como otro, como ontológicamente ajeno, como alteridad radical, y a la vez como la residencia de nuestros posibles" (Batra 2013).

En el departamento de Nariño, los jóvenes rurales identificados como la población económicamente activa podrían visualizar en el ecoturismo oportunidades laborales dado su enfoque en los diferentes componentes como son la investigación, sostenibilidad, producción, asociatividad, transformación, comercialización y modelos socio empresariales incluyentes, buscando nuevas alternativas para generar mayor arraigo en el campo, considerando que el turismo alternativo y sostenible promueve la conservación y el uso racional de los recursos naturales al constituirse no solo como una actividad económica para las comunidades que la ofertan, sino también al considerarse un medio de aprendizaje e interacción constante con el entorno y quienes lo disfrutan como visitantes o excursionistas. De esta manera se pretende sensibilizar y favorecer el desarrollo de actitudes responsables, fomentando una cultura ambiental. Es importante resaltar que el ecoturismo representa una modalidad turística ambientalmente responsable en donde la visita se orienta a espacios naturales con buen estado de conservación, con el propósito es disfrutar, apreciar y estudiar los atractivos naturales y las manifestaciones culturales de dichas áreas (Rojas, 2017). Por lo tanto, las comunidades deben atender las demandas de la sociedad actual presentando una visión integrada de la realidad para comprender que toda acción realizada en cualquier parte afecta al equilibrio general del territorio (Denadić et al., 2016)

En este sentido, el Centro Ambiental Chimayoy es un lugar significativo del territorio departamental, ya que tiene reconocimientos por su potencial ambiental y paisajístico donde la investigación, la conservación y la educación ambiental están reflejadas y vinculadas en las diferentes áreas del centro conformadas por zonas de bosque primario intervenido, secundario, de galerías, en proceso de regeneración natural, con colecciones de plantas aromáticas, medicinales, orquídeas, bromelias y cultivos de labranza mínima (cultivos ancestrales) (López, 2011), lo cual requiere toda la atención para aprovechar la oferta y demanda de estos recursos y servicios ambientales que presta, sin embargo aún es limitada la articulación del mencionado centro con la comunidad aledaña, aspecto que es determinante para consolidar los procesos organizativos y de proyección en el territorio, que facilitaría el desarrollo alternativas de emprendimiento rural, valorando la cultura y tradición. En síntesis, la actual situación del sector rural en Colombia ha generado desplazamientos masivos de la población rompiendo la formación cotidiana adquirida en la familia, provocando la salida de los jóvenes e incorporando nuevas ideas que tienden a desvalorizar lo rural, sumado a ello las limitadas alternativas laborales; por ello, el objetivo de este estudio fue analizar la percepción de jóvenes rurales frente al ecoturismo en el Centro Ambiental Chimayoy, municipio de Pasto, Nariño, por medio del análisis participativo de oportunidades y limitantes frente al ecoturismo y planteamiento de una estrategia de formación ambiental.

\section{METODOLOGÍA}

El Centro Ambiental Chimayoy, Jardín Botánico, administrado por la Corporación Autónoma Regional de Nariño (CORPONARIÑO), se encuentra ubicado en el centro poblado de Daza, corregimiento de Morasurco, municipio de Pasto, departamento de Nariño (Colombia), en el kilómetro. 8 de la vía que conduce de Pasto a Chachagüí, entre los 2736 a los 2955 msnm en el sector noroccidental y su temperatura promedio es de 13 
grados centígrados. Tiene una extensión de 103.5 hectáreas distribuidas en tres veredas. La presente investigación se realizó con una población de 70 jóvenes rurales (mujeres y hombres) que acuden al Centro Ambiental Chimayoy y pertenecen a la Institución Educativa Municipal Morasurco, el muestreo fue de tipo aleatorio simple con estudiantes de grado noveno, décimo y once con un rango de edad entre los 13 y 18 años.

Mediante investigación cualitativa se facilitó la construcción del conocimiento sobre la realidad social y desde la perspectiva de quienes la viven, por lo tanto el proceso metodológico fue adaptado de la investigación de Medina-Cruz et al (2018); es así como primera instancia se realizó un acercamiento y socialización de la investigación y como técnica de recolección de información se diseñó una encuesta semi-estructurada aplicada durante un mes, la cual estuvo conformada por nueve preguntas abiertas y una pregunta de selección múltiple, que dan respuesta a las variables representadas en la tabla 1, permitiendo plantear el diseño de una estrategia para fomentar el ecoturismo en la zona.

Tabla 1: Variables de encuesta para la Identificación percepción jóvenes rurales frente al ecoturismo

\begin{tabular}{|l|l|}
\hline Encuesta & Variables \\
\hline \multirow{3}{*}{$\begin{array}{l}\text { Identificación percepción jóvenes } \\
\text { rurales frente al ecoturismo }\end{array}$} & Interés por la conservación de recursos naturales \\
\cline { 2 - 2 } & Problemas ambientales y sociales de la comunidad \\
\cline { 2 - 2 } & Potencial paisajístico de la zona \\
\cline { 2 - 2 } & Experiencias en ecoturismo \\
\cline { 2 - 2 } & Beneficios del ecoturismo \\
\cline { 2 - 2 } & Impacto del ecoturismo \\
\cline { 2 - 2 } & Actividades de interés relacionadas al ecoturismo \\
\cline { 2 - 2 } & Conocimiento de emprendimiento \\
\hline
\end{tabular}

Además, se utilizaron herramientas participativas como talleres de integración y motivación y un análisis de oportunidades, fortalezas, debilidades y amenazas consideradas por los jóvenes rurales respecto al ecoturismo. La información de la base de datos permitió llevar a cabo un proceso de formación en educación ambiental y ecoturismo, desarrollado con el apoyo interinstitucional para contribuir a la formación de los estudiantes en la temática en mención, con una duración de 12 meses para finalizar con la estructuración una propuesta integral para la zona y varios proyectos formulados por los jóvenes rurales.

\section{RESULTADOS Y DISCUSIÓN}

En la zona de investigación los jóvenes participantes se vincularon por la necesidad de identificar otras opciones que desde su localidad les permita construir su proyecto de vida aprovechando la riqueza ambiental existente con el apoyo institucional.

\section{Identificación de la percepción de jóvenes rurales frente al ecoturismo}

Con relación a la distribución de género, se obtuvo que un $48 \%$ de la muestra equivale a hombres y un $52 \%$ a mujeres que participaron y analizaron la situación del ecoturismo y las distintas problemáticas ambientales y sociales presentes en el corregimiento de Morasurco. El 100\% de los jóvenes manifiesta interés el cuidado de los recursos naturales, manifestando que en la zona aún existen distintos árboles, aves y plantas de tradición para uso medicinal y nacimientos de agua. Considerando que los jóvenes rurales dado su contexto de vida tienen una estrecha conexión con los recursos naturales, que facilita la sensibilización en la importancia de los mismos (Yami et al., 2019), los procesos de formación ambiental contribuyen al análisis de las problemáticas del territorio que permiten generar estrategias de cultura ambiental.

Pese al interés por el cuidado de los recursos naturales, los participantes identifican como problemáticas ambientales la deforestación (62\%), como una situación de alerta, el deficiente uso y manejo de residuos sólidos (33\%) y la contaminación de fuentes hídricas (5\%), situaciones que se presentan por el limitado conocimiento de sus implicaciones en el bienestar de la comunidad. Hernández (2014), describe que el inapropiado saneamiento ambiental es causado por el manejo inadecuado de residuos sólidos, especialmente en su recolección final, generando contaminación ambiental en el corregimiento de Morasurco; sumado a lo anterior, una de las principales problemáticas que se encuentran a lo largo de toda Colombia con relación a los recursos naturales son la deforestación, sedimentación y compactación del suelo que a su vez genera infertilidad en los cultivos, aspectos que tienen una estrecha relación con la afectación antrópica de dichos recursos. 
Referente a las problemáticas sociales que influyen en el cuidado de los recursos naturales, el $73 \%$ considera que existe poco interés, el $14 \%$ es indiferente respecto a la temática, el $8 \%$ resalta que el consumo de drogas afecta su comportamiento y actitud frente a los recursos naturales, el $3 \%$ manifiesta que la escasa comunicación dificulta la organización y gestión, el 1\% opina que son mínimas las oportunidades extra escolares y laborales y el $1 \%$ restante percibe limitantes para acceder a la información sobre temas ambientales que aporten a los jóvenes en su formación. De esta manera Möller (2016), propone como alternativa el ecoturismo, porque permite la inserción de los jóvenes a través del empleo, haciendo posible el arraigo rural y el mantenimiento, conservación y mejora de las zonas naturales. Sin embargo, en este ámbito pueden generarse efectos negativos, por falta de planificación, mala gestión o sobreexplotación de recursos. En cuanto al potencial paisajístico ofertado por el corregimiento de Morasurco, se percibe, que el $73 \%$ de los jóvenes rurales lo mira como un atractivo para los visitantes, teniendo en cuenta su valor ambiental; por otra parte, el $27 \%$ plantean que no se tiene potencial paisajístico, debido al elevado nivel de contaminación ambiental y deterioro de los recursos naturales. El potencial paisajístico puede ser abordado desde una perspectiva visual asociada a sensaciones y recuerdos; dependiendo así de muchas condiciones internas del observador, lo cual podría justificar que los jóvenes se apropian de su territorio y evidencian este de acuerdo a las experiencias que han tenido desde sus propias vivencias en el entorno natural (Murray, 2017).

Respecto a experiencia de ecoturismo, el $78 \%$ manifiesta no tener conocimiento o experiencias; sin embargo, el $9 \%$ lo relaciona con visitas a otros lugares, el $7 \%$ con atractivos turísticos o zonas de interés para visitantes en su región y el $6 \%$ lo considera importante para la sensibilización ambiental. En consideración a lo anterior es de resaltar que en la zona existe un potencial ecoturístico factible de aprovechar desde el Centro Ambiental Chimayoy donde, los jóvenes manifiestan su interés de trabajar en proyectos, con el ánimo de aprender sobre estas temáticas e impartirlas a visitantes, iniciativas de importancia, dado que en la zona no existe mayor oferta de opciones laborales.

Con relación a los beneficios del ecoturismo tan solo el $43 \%$ lo percibe importante, argumentando que podría permitir sensibilización sobre el cuidado de los recursos naturales, el reconocimiento de la zona y la generación de ingresos; el $57 \%$ desconocen los beneficios porque su conocimiento sobre este tema es escaso. Estos resultados son similares a la investigación de Mendoza et al (2013), donde el $54 \%$ de la comunidad resalta el beneficio económico, además del cuidado de los diferentes atractivos y recursos del entorno a través del desarrollo ecoturístico; el cual surge como una alternativa que se caracteriza por realizarse en espacios rurales y basarse en principios de tipo ambiental, social, cultural y económico, de igual manera otros autores exaltan el turismo como una actividad de carácter territorial, que tiene relación directa con las personas, su organización social y cultural, sus vínculos ínterinstitucionales y con el medio ambiente (Schismenos et al., 2018; Bailey, 2016).

En cuanto al conocimiento sobre el impacto del ecoturismo en el territorio, el $72 \%$ de los jóvenes rurales no tienen claridad al respecto, sin embargo, para el $27 \%$ el impacto es positivo porque permite mayor sensibilización ambiental y el $1 \%$ menciona que se genera impacto cuando se adecuan zonas recreativas. De esta manera la protección de las áreas naturales y la sensibilización, contribuye con iniciativas o proyectos que impulsen la recuperación de plantas endémicas, la medicina tradicional, el manejo de los residuos y el cuidado de los recursos naturales, entre otros (Cini y Passafaro, 2019). Dentro de las actividades ecoturísticas, los jóvenes rurales tuvieron mayor claridad para relacionarlo con sus vivencias personales, donde las actividades más llamativas fueron: la observación de fauna y flora (25\%), la caminata guiada (23\%) y los talleres de educación ambiental (21\%). Pariente et al. (2016), evidencia resultados similares en su investigación resaltando que las actividades turísticas de mayor relevancia fueron los paseos campestres y la observación de aves, también para Howitt y Mason (2018) son el senderismo y el agroturismo.

En consideración al conocimiento sobre emprendimiento, el $56 \%$ de los jóvenes no tiene claridad al respecto y desconoce las ventajas que podrían beneficiarlos, talvez, influenciados por factores sociales, económicos y ambientales de su entorno, que reducen las alternativas visionarias en su propio territorio. Según Villota (2017), en su investigación en instituciones educativas rurales del municipio de Pasto, encontró que el 38\% de los estudiantes en alguna ocasión han puesto en práctica una idea de negocio, entendida como el producto o servicio que se quiere ofrecer al mercado y por la cual recibe una retribución económica, donde la mayoría manifiesta encontrarse en una etapa inicial; por otra parte, el $62 \%$ de los estudiantes no han tenido o puesto en práctica emprendimientos, situación que amerita atención, toda vez que éste tipo de experiencias afianzan sus capacidades y hacen parte de su formación integral para afrontar su futuro desempeño laboral, aspectos acordes a lo planteado por Jones y Spadafora, 2016; Acosta y Díaz, 2018, quienes plantean que el ecoturismo un atractivo para las nuevas generaciones, quienes participan y aprenden de la cultura y del entorno natural resaltando su valor e importancia en acciones de emprendimiento. 


\section{Análisis de oportunidades y limitantes del ecoturismo identificado por jóvenes rurales}

Mediante el análisis situacional, se identificó de manera participativa la percepción de los jóvenes rurales con relación a las debilidades, fortalezas, oportunidades y amenazas del desarrollo ecoturístico en el Centro Ambiental Chimayoy, expuesto en la tabla 1. Es importante resaltar que los procesos de planificación participativa para el desarrollo de propuestas ecoturísticas en el Centro Ambiental Chimayoy con la comunidad y en este caso con jóvenes rurales, es una de las alternativas para planificar el ecoturismo en un destino, haciendo referencia a la actividad que se fundamenta en la creación de productos turísticos bajo el principio básico de la necesaria participación de la comunidad local, y donde se pretende reducir el impacto negativo y reforzar los impactos positivos del turismo en la naturaleza (Khoshtaria y Chachava, 2017).

Tabla 1: Análisis situacional de ecoturismo identificado por jóvenes rurales

\begin{tabular}{|c|c|}
\hline Debilidades & Fortalezas \\
\hline $\begin{array}{l}\text { Falta de organización social } \\
\text { Inexistencia de juntas de acción comunal }\end{array}$ & $\begin{array}{l}\text { Gran porcentaje de los jóvenes cuenta con } \\
\text { educación escolar }\end{array}$ \\
\hline $\begin{array}{l}\text { Bajo nivel de educación ambiental y cuidado del } \\
\text { medio ambiente }\end{array}$ & $\begin{array}{l}\text { Interés en proponer soluciones innovadoras a la } \\
\text { problemática local }\end{array}$ \\
\hline $\begin{array}{l}\text { Desinterés en temáticas relacionadas con el medio } \\
\text { ambiente }\end{array}$ & $\begin{array}{l}\text { Conocimiento ancestral sobre su localidad Riqueza } \\
\text { en flora y fauna }\end{array}$ \\
\hline Oportunidades & Amenazas \\
\hline \multirow{3}{*}{$\begin{array}{l}\text { Apoyo institucional en el corregimiento } \\
\text { Acceso a la educación } \\
\text { Oferta laboral cercana a la ciudad }\end{array}$} & $\begin{array}{l}\text { Desastres naturales debido a la cercanía del volcán } \\
\text { Galeras }\end{array}$ \\
\hline & \\
\hline & $\begin{array}{l}\text { Contaminación generada por empresas } \\
\text { prestadoras de servicios como Montagas y Texaco }\end{array}$ \\
\hline
\end{tabular}

El ecoturismo tiene varios objetivos como: la conservación de los recursos naturales, patrimoniales y culturales; el desarrollo socioeconómico de las comunidades locales; y la calidad recibida por la demanda turística en el destino geográfico (Denadić et al., 2016). Por tanto, es una actividad económica que puede fomentar el desarrollo socioeconómico, cultural y medioambiental de un destino, pero que requiere de una planificación adecuada, que tiene como finalidad controlar las posibles desviaciones provocadas por esta actividad, que pueden transformarse en impactos negativos para las comunidades locales, donde la percepción local y el análisis situacional son insumos para la generación de propuestas acordes a las necesidades comunitarias (Ocampo y Winton, 2017).

\section{Estrategias para fomentar el ecoturismo}

Mediante la educación ambiental se pueden romper los paradigmas que no se adecuan a la nueva realidad y transmitir nuevos valores a los educandos, cambiando las relaciones con sí mismos, con los demás y con la naturaleza. Así se plantará una semilla de responsabilidad en cada uno de ellos para que puedan transformar sus acciones cotidianas en experiencias capaces de generar actos locales para resolver problemas globales (Khattab y Haggar, 2015). En consideración a lo anterior de manera participativa se plantearon dos estrategias para fomentar el ecoturismo 1) formación en educación ambiental y ecoturismo y 2) formulación de propuestas ecoturísticas de jóvenes rurales y una propuesta integral

\section{Formación en educación ambiental y ecoturismo}

Con el análisis obtenido de este estudio se planteó como estrategia de formación la estructuración de un diplomado con el objetivo de fortalecer y desarrollar las capacidades de los jóvenes rurales en procesos de educación ambiental y ecoturismo en el marco de la interacción con el Centro Ambiental Chimayoy y la gestión interinstitucional. A través de diferentes herramientas conceptuales, metodológicas y prácticas se orientó la comprensión del territorio desde un escenario de paz brindando la oportunidad de capacitarse en alternativas de emprendimiento rural. Con el diplomado se beneficiaron 25 jóvenes rurales quienes cursaron 5 módulos de capacitación en temáticas como historia, patrimonio y biodiversidad; educación ambiental para el ecoturismo; factores de evaluación de atractivos turísticos; emprendimiento innovador y componentes de sostenibilidad. El enfoque de esta formación se orientó hacia la construcción del conocimiento a partir de la reflexión sobre el medio rural y su cotidianidad para proponer alternativas de mejoramiento pertinentes.

Entre las herramientas didácticas empleadas en la formación del diplomado se destacan: conferencias; talleres como instrumento estratégico para el desarrollo de situaciones educativo - ambientales y de construcción de conocimiento para la interpretación y comprensión de la realidad ambiental; mesas de trabajo 
para debate y análisis; estudio de caso práctico para la implementación de espacio fotográfico como atractivo turístico en el Centro Ambiental Chimayoy. Lo anterior es similar a lo desarrollado en comunidades indígenas de Nicaragua, quienes ofrecieron un diplomado en economías comunitarias, el cual surgió como resultado del diagnóstico de necesidades de formación, logrando establecer un componente de formación de talentos humanos indígenas como parte del proyecto de fortalecimiento en la edificación de la autonomía enfocándose hacia el ecoturismo como alternativa (López y Calero, 2018).

\section{Propuestas ecoturísticas formuladas por jóvenes rurales}

Como resultado del proceso de investigación en el presente estudio, los jóvenes rurales lograron obtener bases conceptuales que les permitió formular sus propuestas sobre ecoturismo y educación ambiental, consideradas como iniciativas innovadoras para empezar a gestionar proyectos a mediano plazo e implementarlos en su región. En la tabla 2 se sintetiza la información sobre cada una de las propuestas elaboradas. Alcanzar la consolidación de ideas en proyectos como primeras iniciativas de jóvenes, es significativo para la motivación de ciudadanos responsables con el ambiente. A través de la interacción entre teoría y práctica, se logra la formación de talentos, la recopilación de un material básico de información y de análisis, del cual surgen diferentes proyectos estratégicos para el desarrollo de las comunidades, es así como López y Calero (2018), obtuvieron 15 proyectos, los cuales son el resultado del análisis detallado de la información que se obtuvo del diagnóstico social, económico y ambiental en los pueblos indígenas que estudiaron y el apoyo a los mismos para generar iniciativas que los beneficien.

Tabla 2: Propuestas formuladas por jóvenes rurales para fomentar ecoturismo en el Centro Ambiental Chimayoy

\begin{tabular}{|l|l|}
\hline Titulo & Descripción \\
\hline Manchakuy & $\begin{array}{l}\text { Selección estratégica de un sendero dentro de las instalaciones del Centro Ambiental } \\
\text { Chimayoy, que se caracterizará por la existencia de vegetación densa que brinde un } \\
\text { ambiente propicio para recorridos temáticos, donde los visitantes tendrán la posibilidad de } \\
\text { escuchar relatos sobre las leyendas que más impacto han tenido en el corregimiento de } \\
\text { Morasurco. }\end{array}$ \\
\hline Arte con flores & $\begin{array}{l}\text { Adecuación de un espacio para la siembra de plantas ornamentales en diseños creativos } \\
\text { de distintas figuras artísticas, teniendo en cuenta la cultura narinense y colombiana, } \\
\text { representando la biodiversidad de la zona altoandina. }\end{array}$ \\
\hline Vida fotográfica & $\begin{array}{l}\text { Registro de diversas especies de flora nativas del corregimiento de Morasurco presentes } \\
\text { en el Centro Ambiental Chimayoy para sensibilizar sobre la importancia en la conservación } \\
\text { de los ecosistemas estratégicos. }\end{array}$ \\
\hline Sendero de la vida & $\begin{array}{l}\text { Adecuación de senderos con mensaje ambientales elaborados con material reciclado para } \\
\text { sensibilizar a visitantes respecto a la conservación de los recursos naturales de la región. }\end{array}$ \\
\hline $\begin{array}{l}\text { Avistamiento de } \\
\text { aves }\end{array}$ & $\begin{array}{l}\text { Formación de guías para identificar la diversidad de especies de aves y orientar en su } \\
\text { conocimiento a los visitantes. }\end{array}$ \\
\hline Mejora tu salud & $\begin{array}{l}\text { Oferta de recorridos agroturísticos por parcelas de producción de aromáticas, rescatando } \\
\text { saberes locales de cultivo y aprovechamiento de plantas medicinales. }\end{array}$ \\
\hline Actívate & $\begin{array}{l}\text { Adecuación de un circuito de } 5 \text { kilómetros para entreno y eventos competitivos de atletismo } \\
\text { a campo traviesa en sitios estratégicos del Centro Ambiental Chimayoy, coordinado por un } \\
\text { equipo de jóvenes rurales capacitados. }\end{array}$ \\
\hline
\end{tabular}

\section{Propuesta integral}

Actualmente las actividades antrópicas como la deforestación, agricultura convencional, mala disposición de desechos, ganadería, contaminación de fuentes hídricas; además de las exigencias económicas de la sociedad y la reducida formación ambiental, han generado fuertes impactos en los recursos naturales donde una consecuencia es el cambio climático que ha repercutido en las comunidades a nivel social, económico y ambiental (Acosta y Díaz, 2018). Es así como la educación ambiental surge de la necesidad por concientizar a los seres humanos en la preservación de la naturaleza, recursos naturales y soluciones a problemas ambientales.

En el departamento de Nariño la implementación del Plan Departamental Decenal de Educación Ambiental, (2010), que tiene como visión "instalar marcos conceptuales, contextuales y proyectivos, que permitan la orientación procesal y estratégica, de la incorporación de la educación ambiental, hacia la generación de cambios en los sistemas comportamentales y de valores, para la construcción y gestión de una cultura ambiental ética y responsable en el manejo sostenible del ambiente, con participación interinstitucional, intersectorial, comunitaria y étnica". De igual manera la Corporación Autónoma Regional de Nariño - 
CORPONARIÑO, en el Plan de Gestión Ambiental Regional del departamento de Nariño, PGAR (2016:188), ha contemplado en los procesos de educación ambiental la consolidación de una estrategia de educación ambiental enfocada a promover un cambio de actitudes y comportamientos en los habitantes de la zona de influencia, a través la valoración de la biodiversidad y los ecosistemas.

Es así, como el Centro Ambiental Chimayoy administrado por CORPONARIÑO, es un espacio significativo del territorio departamental, ya que tiene reconocimiento por su potencial ambiental, recreacional y paisajístico. Aunque la educación ambiental es una prioridad dentro del centro, principalmente por las actividades ecoturísticas, aún no se ha evaluado cómo el ecoturismo puede contribuir a los procesos de formación ambiental. En la tabla 3, se presenta la estructura general de una propuesta integral como estrategia para fomentar el ecoturismo y la educación ambiental.

Tabla 3: Propuesta integral: Estrategia pedagógica de educación ambiental en el Centro Ambiental Chimayoy

\begin{tabular}{|l|l|}
\hline Ítem & Descripción \\
fermulación problema & $\begin{array}{l}\text { ¿Puede ser el ecoturismo una estrategia pedagógica de educación ambiental en el Centro } \\
\text { Ambiental Chimayoy? }\end{array}$ \\
\hline Objetivos & $\begin{array}{l}\text { Objetivo General: Evaluar el ecoturismo como una estrategia pedagógica de educación } \\
\text { ambiental en el Centro Ambiental Chimayoy, municipio de Pasto, Nariño. } \\
\text { Objetivos Específicos: a) Diagnosticar los procesos ecoturísticos que se desarrollan en el } \\
\text { Centro Ambiental Chimayoy.; b) Estructurar una propuesta de ecoturismo como estrategia } \\
\text { pedagógica para la educación ambiental en el Centro Ambiental Chimayoy. }\end{array}$ \\
\hline Metodología & $\begin{array}{l}\text { Mediante Investigación Acción Participativa, se pretende fortalecer redes colaborativas } \\
\text { para la integración del talento humano en el planteamiento de estrategias pedagógicas } \\
\text { pertinentes para examinar la forma en que los individuos perciben y experimentan los } \\
\text { fenómenos que los rodean, profundizando en sus puntos de vista, interpretaciones y } \\
\text { significados. } \\
\text { Como actividades a desarrollar se plantean: a) socialización del proyecto de investigación; } \\
\text { b) diseño e implementación de encuesta para diagnosticar el ecoturismo en el Centro } \\
\text { Ambiental Chimayoy; c) Realización de talleres diagnósticos participativo estimación de } \\
\text { capacidad de carga para senderos; d) Análisis de información y planteamiento de } \\
\text { propuestas innovadoras ligadas al ecoturismo como estrategia de educación ambiental; e) } \\
\text { Validación y ajuste de propuesta de ecoturismo como estrategia pedagógica de educación } \\
\text { ambiental }\end{array}$ \\
\hline $\begin{array}{l}\text { Resultados } \\
\text { esperados }\end{array}$ & $\begin{array}{l}\text { Diagnóstico del ecoturismo en el Centro Ambiental Chimayoy } \\
\text { Propuesta de ecoturismo como estrategia pedagógica de educación ambiental } \\
\text { Base de datos sobre capacidad de carga en senderos }\end{array}$ \\
\hline
\end{tabular}

\section{CONCLUSIONES}

El ecoturismo brinda la alternativa y herramientas idóneas para conectar la realidad social de los jóvenes rurales con los procesos de desarrollo de la localidad, donde el Centro Ambiental Chimayoy es estratégico para potenciar las habilidades de liderazgo, fortalecimiento de la cultura y estrechar lazos con su territorio, fomentando la nueva ruralidad. Para iniciar procesos de formación, es necesario conocer la percepción de las comunidades y su visión frente al desarrollo de su comunidad, de esta manera se facilita consolidar estrategias ecoturísticas dinámicas, atractivas y motivadoras para que las nuevas generaciones sean gestoras de su propio desarrollo, de ahí la importancia del apoyo interinstitucional para conjuntamente lograr proyectos que vinculen la academia al sector productivo y mediante la corresponsabilidad se propenda por emprendimientos innovadores.

Se evidencia la necesidad de formación pertinente, más aún en el contexto de vida rural, cuya dinámica conlleva a redimensionar la conexión directa con el ambiente, situación que amerita estudios interdisciplinares e interinstitucionales para contribuir en la orientación de las nuevas generaciones para que sean capaces de afrontar los desafíos ambientales asumiendo con responsabilidad su compromiso; con la propuesta planteada como resultado del estudio, se espera desarrollar una experiencia ecoturística como estrategia pedagógica en el Centro Ambiental Chimayoy, cuya implementación será participativa para generar empoderamiento de la comunidad y que está sea la gestora de una cultura ambiental 


\section{REFERENCIAS}

Acosta, S. y Diaz, F., Strategy for the Sustainable use of Ecotourism in the Route of the Colombian Piedemonte. https: //doi.org/10.14207/ejsd.2018.v7n3p225., European Journal of Sustainable Development, 7(3), 225-236, (2018).

Alvarado S. V., Martínez, J. E. y Muñoz, D. A., Contextualización teórica al tema de las juventudes: una mirada desde las ciencias sociales a la juventud. Revista Latinoamericana de Ciencias Sociales, Niñez y Juventud. Manizales, 7(1), 83- 102 (2009).

Bailey, J., Ecotourism Social Enterprise: Learning From the Global South, 1a edición, Capstone Collection, Washington, DC, USA (2016).

Batra, A., Ceceña, A.E. y otros tres autores., Crisis civilizatoria y superación del capitalismo, 1a Ed., 218, Instituto de Investigaciones Económicas (2013).

Cini, F y Passafaro, P., Youth and ecotourism: A qualitative exploration, Tourism and Hospitality Research, https://doi.org/10.1177/1467358417704887, 19 (1), 126 a 131 (2019).

Corporación Autónoma Regional de Nariño., Plan de Gestión Ambiental Regional del Departamento de Nariño - PGAR 2016 - 2036. (2016).

Corporación Autónoma Regional de Nariño., Plan Decenal de Educación Ambiental - PDEA 2010 - 2020. Pasto, Nariño, (2010).

Denadić, M., Muhi, B, y Jovanović, D. Rural Tourism Serbia's Missed Chance, https://doi.org/10.5937/ekoPolj1602515D, Economics of Agriculture, 63(2), 515 a 529 (2016).

Heidkamp C.P y Morrissey, J. Towards coastal resislience and sustainability. $1^{a}$ edición, Routledge, (2019).

Hernández, G., Salud e infraestructura sanitaria en los corregimientos de Pasto, Revista UNIMAR; 28(1), 9 a 14 (2014).

Howitt, J y Mason, C.W., Ecotourism and sustainable rural development in Pérez Zeledón, Costa Rica. The Journal of Rural and Community Development, 13(1), 67 a 84 (2018).

Jones, G y Spadafora, A., Entrepreneurs and the Co Creation of Ecotourism in Costa Rica, Harvard Business school, $1^{\text {a }}$ edición, 1 a 56 (2016).

Khattab, M y Haggar, S., A Novel Approach Towards a Sustainable Ecotourism Community, Int. J. of Sustainable Water \& Environmental Systems, 7(1), 9 a 19 (2015).

Khoshtaria, T.K y Chachava, N.T., Prospects of ecotourism development in recreation areas of South Georgia. https://doi.org/10.1016/j.aasci.2017.07.004, Annals of Agrarian Science, 15(3), 312 a 317 (2017).

Leff, E., Ética por la Vida. Elogio de la Voluntad de Poder. POLIS, Revista Latinoamericana, 5(13), 1 - 32, (2006).

López, E.C y Calero, W.A., Acompañamiento en procesos de formación a jóvenes líderes de pueblos indígenas del Pacífico Centro Norte de Nicaragua. https://doi.org/10.30698/recsp.v1i2.14, Revista electrónica de conocimientos, saberes y prácticas, 1(2), 83 a 90 (2018).

López, M.A., Determinación de herramientas básicas para la ordenación del uso público en el Centro Ambiental Chimayoy, San Juan de Pasto, Colombia. Trabajo de grado para el título de magister en Ciencia. Universidad internacional de Andalucía, 136, (2011).

Medina-Cruz, H., Lagunes-Domínguez, A. y Torres-Gastelú, C.A., Percepción de estudiantes de nivel de secundaria sobre el uso de las TIC en su clase de ciencias, Información tecnológica, 29(4), 259 - 266 (2018).

Mendoza, M.M., Rodríguez, G. y Enciso, M.J., Actitud de la comunidad local como factor de éxito en un proyecto turístico. Revista de cultura y turismo, 7(1), 4 a 30 (2013).

Murray, A.E., Footprints in paradise, ecotourism, local knowledge, nature therapies in Okinawa, $1^{\text {a }}$ edición, 1 a 170 , Berghahn Books, New York, (2017).

Möller, P., Young adults' perceptions of and affective bonds to a rural tourism community. International Journal of Geography, 194(1), $32-45,(2016)$.

Neleman, S. y Castro, F., Between nature and the city: youth and ecotourism in an Amazonian 'forest town' on the Brazilian Atlantic Coast, https://doi.org/10.1080/14724049.2016.1192181, Journal of Ecotourism, 15(3), 261 a 284, (2016).

Ocampo, N. y Winton, S., Economic and Conservation Potential of Bird Watching Tourism in Postconflict Colombia, https://doi.org/10.1177/1940082917733862, Tropical Conservation Science, 10(1), 1 a 6, (2017).

Pariente, E., Chávez, J. y Reynel, C., Evaluación de potencial turístico del distrito de Huarango, San Ignacio, Cajamarca, Perú, http://dx.doi.org/10.21704/rea.v15i1.581, Ecología aplicada, 15(1), 37 a 46 (2016).

Pigem, J., Crítica de la (Ir) racionalidad económica. Revista Mediterráneo Económico. 23 (1), 157 - 168 (2013).

Rojas, R, La planeación del turismo en áreas naturales protegidas: Valle de los Cirios, Baja California, México. En libro: Repensando el turismo sustentable, 1a Ed, Universidad Autónoma del Estado de México, 213 - 236, México, (2017). 
Schismenos, S., Zaimes, G. y otros dos autores. Environmental sustainability and ecotourism of riparian and deltaic ecosystems: opportunities for rural Eastern Macedonia and Thrace, Greece,

https://doi.org/10.1080/00207233.2018.1510579, International Journal of Environmental Studies, (2018).

Toledo, V.M., La perspectiva etnoecológica. Cinco reflexiones acerca de las ciencias campesinas sobre la naturaleza con especial referencia a México. Revista Ciencias 4(1),22 - 29, (1990).

Villota, C.D., Caracterización de la cultura del emprendimiento en las Instituciones de Educación Municipal del sector rural del municipio de Pasto, Trabajo de grado para master en administración de organizaciones, Universidad Nacional Abierta y a Distancia, Pasto, 141 (2017).

Yami, M., Feleke, S. y otros cuatro autores, African Rural Youth Engagement in Agribusiness, Achievements, Limitations, and Lessons, https://doi.org/10.3390/su11010185, Sustainability, 11(1), 1 a 5 (2019). 
